\title{
QUANTILE-ORIENTED GLOBAL SENSITIVITY ANALYSIS OF DESIGN RESISTANCE
}

\author{
Zdeněk KALA(10* \\ Department of Structural Mechanics, Faculty of Civil Engineering, Brno University of Technology, \\ Veveři 95, 602 00, Brno, Czech Republic
}

Received 09 January 2019; accepted 24 January 2019

\begin{abstract}
The article investigates the application of a new type of global quantile-oriented sensitivity analysis (called QSA in the article) and contrasts it with established Sobol' sensitivity analysis (SSA). Comparison of QSA of the resistance design value ( 0.1 percentile) with SSA is performed on an example of the analysis of the resistance of a steel IPN 200 beam, which is subjected to lateral-torsional buckling. The resistance is approximated using higher order polynomial metamodels created from advanced non-linear FE models. The main, higher order and total effects are calculated using the Latin Hypercube Sampling method. Noticeable differences between the two methods are found, with QSA apparently revealing higher sensitivity of the resistance design value to random input second and higher order interactions (compared to SSA). SSA cannot identify certain reliability aspects of structural design as comprehensively as QSA, particularly in relation to higher order interactions effects of input imperfections. In order to better understand the reasons for the differences between QSA and SSA, two simple examples are presented, where QSA (median) and SSA show a general agreement in the calculation of certain sensitivity indices.
\end{abstract}

Keywords: sensitivity analysis, quantile, resistance, lateral-torsional buckling, imperfections, steel, random sampling.

\section{Introduction}

The fundamental characteristic of safety and reliability of the design of load bearing structures is the design value of resistance (Galambos, 1998). In order to verify if a structure can bear the relevant loadings, referred to as the ultimate limit state, it is necessary to check if the resistance capacity is equal to or greater than the sum of the relevant action effects (Sedlacek \& Müller, 2006; Sedlacek \& Kraus, 2007).

Standard EN 1990:2002 (2003) provides a semi-probabilistic procedure for the safety assessment of design methods. Design methods can be verified by comparing the design values contained in the Eurocodes with the design quantiles obtained using stochastic analysis. For example, the design resistance of a steel structure calculated according to Eurocode 3 (EN 1993-1:2005, 2005) should correspond approximately to $0.1 \%$-quantile of the random resistance $R$ (Kala, 2012). The random variability of $R$ can either be identified on the basis of physical experiments performed in the laboratory, or these experiments can be simulated on a computer using a stochastic computational model with consideration to the effects of all important in- itial imperfections (see, e.g. Vales \& Stan, 2017; Jönsson \& Stand, 2017; Liu, He, Yhenyu, \& Yuan, 2018).

The approaches described in design standards often require further explanation for a full understanding of their background in order to reduce the possibility of errors. The fundamental question is which initial imperfections ("imperfections") are crucial, in the sense that their random variability significantly affects the design resistance? The motivation for work presented is to analyze the sensitivity of the resistance design value (design quantile) to random input initial imperfections. The statistical characteristics of these imperfections must then be determined with high precision.

Common sensitivity analysis methods (Saltelli, Chan, \& Scott, 2004) monitor the correlation between model inputs and the output or the effects of random inputs on the variance of model output. However, these methods may not be suitable for analysing the effects of random imperfections on the design quantile $R_{d}$. In accordance with the classical utility theory, variance is not sufficient for the determination of the decision-maker state of knowledge

*Corresponding author. E-mail: kala.z@fce.vutbr.cz 
in general (Borgonovo, 2007; Antucheviciene, Kala, Marzouk, \& Vaidogas, 2015). This problem can be partially overcome using sensitivity analysis based on factorial design (SAFD), which describes the influence of inaccuracies in the statistical moments of input imperfections on the $R_{d}$ (Kala \& Valeš, 2017a, 2017b).

The so-called Goal Oriented Sensitivity Analysis, which uses new global sensitivity indices subordinated to contrast functions, is more general in use (Fort, Klein, \& Rachdi, 2016). The choice of the contrast function can determine global sensitivity indices of different types.

Contrast functions are an important part of the statistical learning theory (Vapnik, 1998; Massart, 2003) where they define estimation procedures of certain features, which are associated to a random model output. Quantilecontrast functions, which measure the loss (distance) between $R$ and $R_{d}$ in dependence to the influence of input random imperfections, are used in the article. Loss has the significance of contrast because it highlights the characteristics of numerical models involved in the used statistical approach (Rachdi, 2011).

The quantile-contrast function measures the absolute distance (contrast) between $R$ and $R_{d}$. The other contrast functions are defined in Fort et al. (2016). It has been shown in Fort et al. (2016) that use of the quadratic contrast function with the mean minimiser leads to established Sobol' sensitivity indices (Sobol', 1993, 2001). The first order quantile contrast index has been studied and applied in Browne, Fort, Iooss, and Gratiet (2017) and Maume-Deschamps and Niang (2018). The measures proposed in Kucherenko, Song, and Wang (2019) make use of the mean distance between quantiles rather than the mean distance between average contrast functions as in the case of quantile-oriented sensitivity indices. The first and higher order quantile contrast indices have been compared with Sobol' global sensitivity analysis in an example using Ishigami Function in Kala (2018).

The aim of the present article is global quantile-oriented sensitivity analysis (QSA) of $R_{d}$ performed by numerical estimation of all first and higher order quantile contrast indices. QSA has a high potential to be developed for the analysis of design structural reliability based on stochastic models and design quantile (see, e.g. D’Angelo \& Nussbaumer, 2017; Kala, Valeš, \& Jönsson, 2017; Chalmovsky et al., 2017; Hariri-Ardebili \& Pourkamali-Anaraki, 2018). Theoretical development of QSA for models with correlated inputs can be expected analogously to the development of Sobol' sensitivity analysis (SSA) (see, e.g. K. Zhang, Lu, Wu, \& Y. Zhang, 2017; Li, Lu, Zhang, \& Gao, 2017; Xiao, Lu, \& Wang, 2018).

The numerical example presented in this article builds on Kala and Valeš (2017a, 2017b), describing in detail the advanced non-linear FE model of a steel beam, which is subjected to lateral-torsional buckling (LTB) due to uniform bending moment. Resistance $R$ (model output) is denoted as LTB-R (Kala \& Valeš, 2017b) or LCC (Kala \& Valeš, 2017a) in previous studies. SSA of $R$ published in Kala and Valeš (2017b) is extended to beams of higher strength class and compared with QSA of $R_{d}$.

The noticeable differences in the results of QSA and SSA are found and their effects on structural reliability are discussed. QSA revealing higher sensitivity of the resistance design value to random input higher order interactions (compared to SSA).

\section{Global quantile-oriented sensitivity analysis}

Given a model of the form $Y=f\left(X_{1}, X_{2}, \ldots, X_{M}\right)$, where $Y$ is a scalar output and $X_{i}$ are $M$ statistically independent input variables. The contrast function $\psi$ associated with $\alpha$-quantile can be written with parameter $\theta$ as

$$
\psi(\theta)=E(\psi(Y, \theta))=E\left((Y-\theta)\left(\alpha-1_{Y<\theta}\right)\right)
$$

and the estimator of $\alpha$-quantile $\theta^{*}$ is given by $\theta^{*}=$ Argmin $\psi(\theta)$. Based on Fort et al. (2016), the first order quantile contrast index $Q_{i}$ (first order or main sensitivity index subordinated on the contrast) can be defined as

$$
Q_{i}=\frac{\min _{\theta} \psi(\theta)-E\left(\min _{\theta} E\left(\psi(Y, \theta) \mid X_{i}\right)\right)}{\min _{\theta} \psi(\theta)},
$$

where the numerator is the contrast variation due to $X_{i}$. $Q_{i}$ is the sensitivity index of the estimator of $\theta^{*}$. The minimum value of (1) can be calculated, for e.g., using $K$ runs of the Latin Hypercube Sampling method (LHS) (McKey, Beckman, \& Conover, 1979; Iman \& Conover, 1980) as

$$
\begin{aligned}
\min _{\theta} \psi(\theta) \approx \frac{1}{K} \sum_{k=1}^{K} & \left(f\left(X_{1}(k), X_{2}(k), \ldots, X_{M}(k)\right)-\theta^{*}\right) \\
& \left(\alpha-1_{f}\left(X_{1}(k), X_{2}(k), \ldots, X_{M}(k)\right) \leq \theta^{*}\right),
\end{aligned}
$$

where $\theta^{*}$ is the estimator of $\alpha$-quantile. The $\alpha$-quantile $\theta^{*}$ can be estimated from LHS runs so that $\alpha \times K$ runs of $Y$ are smaller than $\theta^{*}$ and $(1-\alpha) \times K$ runs of $Y$ are greater than $\theta^{*}$, see the example in Figure 1. The estimate of 0.001 -quantile is practically evaluated as the $400^{\text {th }}$ smallest value in the set arranged in ascending order of $K=400000$ runs of LHS, see Figure 1.

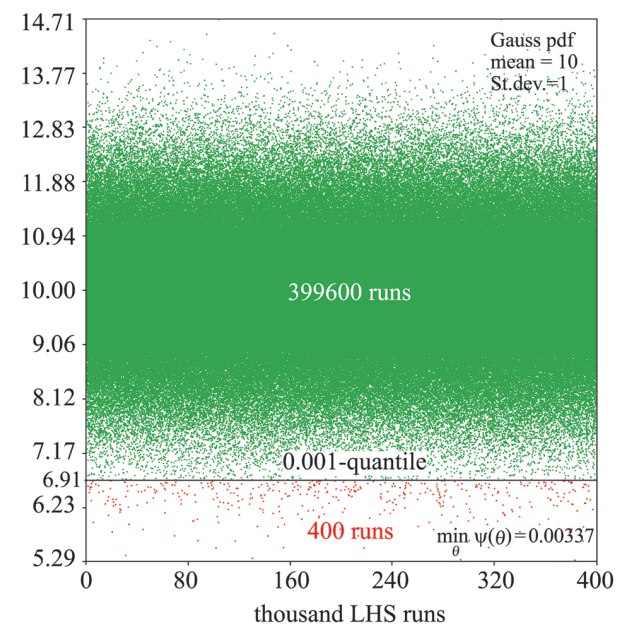

Figure 1. Example of the estimate of the 0.001-quantile from 400000 LHS runs $101 \times 104 \mathrm{~mm}(300 \times 300 \mathrm{DPI})$ 
The second member in the numerator (2) can be calculated using two sets of the LHS method. $N$ random realizations of $X_{i}$, i.e. $X_{i}(1), \ldots, X_{i}(j), \ldots, X_{i}(N)$ are generated in the first set. Then, $K$ random realizations of vector $X_{\sim i}$ are generated for each realization $X_{i}(j), j=1, \ldots, N$ (all variables but $\left.X_{i}\right)$. For fixed $X_{i}$ we can calculate

$$
\begin{array}{r}
\min _{\theta} E\left(\psi(Y, \theta) \mid X_{i}\right) \approx m(j)=\frac{1}{K} \sum_{k=1}^{K}\left(f\left(X_{i}(j), X_{\sim i}(j, k)\right)-\right. \\
\left.\theta^{*}(j)\right)\left(\alpha-1_{\left.f\left(X_{i}(j), X_{\sim i}(j, k)\right) \leq \theta^{*}(j)\right),}\right.
\end{array}
$$

where conditional $\alpha$-quantile $\theta^{*}(j)$ is calculated in a similar manner as in (3) with the difference that the runs of $Y$ are obtained for $K$ random realizations of variables $X_{\sim i}$ and fixed (non-random) $X_{i}$. For $N$ runs of $X_{i}$ we subsequently obtain

$$
E\left(\min _{\theta} E\left(\psi(Y, \theta) \mid X_{i}\right)\right)=\frac{1}{N} \sum_{j=1}^{N} m(j) .
$$

The second order quantile contrast index $Q_{i j}$ is defined as

$$
Q_{i j}=\frac{\min _{\theta} \psi(\theta)-E\left(\min _{\theta} E\left(\psi(Y, \theta) \mid X_{i}, X_{j}\right)\right)}{\min _{\theta} \psi(\theta)}-Q_{i}-Q_{j} \text {. }
$$

The higher order quantile contrast indices can be expressed analogously. The sum of all sensitivity indices must be equal to one:

$$
\sum_{i} Q_{i}+\sum_{i} \sum_{j>i} Q_{i j}+\sum_{i} \sum_{j>i} \sum_{k>j} Q_{i j k}+\ldots+Q_{123 \ldots M}=1 .
$$

It can be noted that the use of the contrast function

$$
\psi(\theta)=E(Y-\theta)^{2}
$$

transforms (1), (2), (6) and (7) to the classical Sobol' decomposition (Sobol', 1993, 2001), in which the first order sensitivity index $S_{i}$ is defined as

$$
\begin{gathered}
S_{i}=\frac{V(Y)-E\left(V\left(Y \mid X_{i}\right)\right)}{V(Y)}=\frac{V\left(E\left(Y \mid X_{i}\right)\right)}{V(Y)}= \\
\operatorname{corr}^{2}\left(Y, E\left(Y \mid X_{i}\right)\right),
\end{gathered}
$$

where corr is Pearson correlation coefficient. Sobol' higher order sensitivity indices are calculated in a similar manner, see (e.g. Saltelli et al., 2004).

\section{Basic research examples}

QSA and SSA are based on the decomposition of the response function. However, each method uses a different contrast function. QSA measures the contrast as the absolute distance from the conditional quantile (1), while SSA measures the contrast as the square of the distance from the conditional arithmetic mean (8). Each sensitivity analysis has a different descriptive (measuring) capability. Two examples are presented, where QSA and SSA exhibit partial or general agreement, to enable better understanding of the reasons that lead to differences between QSA and SSA.

\subsection{Additive model}

Let us consider the linear function

$$
Y=x_{1}+x_{2}+x_{3},
$$

where $x_{1}, x_{2}, x_{3}$ are statistically independent random variables. SSA evaluates the model (10) as perfectly additive without input interactions. The aim of the study is to find such input pdf so that there is an agreement in sensitivity measurements of both QSA and SSA, i.e. without interaction effects.

Let us consider input variables $x_{i}(i=1,2,3)$ with twopoint probability mass function (pmf), see Figure 2. All the values of the pmf must be non-negative and sum up to 1 and $a \neq b$. Then QSA and SSA yield the same results when $p \geq 0.8$ or $p \leq 0.2$. The results are only first order sensitivity indices $S_{1}=S_{2}=S_{3}=Q_{1}=Q_{2}=Q_{3}=0.333$. All higher order indices are zero.

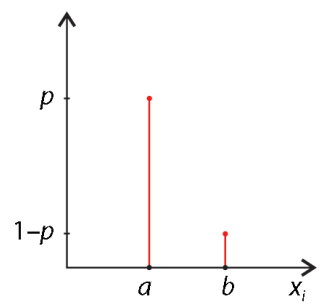

Figure 2 . The probability mass function $36 \times 34 \mathrm{~mm}$ $(300 \times 300 \mathrm{DPI})$

The agreement between QSA and SSA can be clearly illustrated on an example with $p=0.8, a=0, b=1$ solved using the LHS method. The 0.5-quantile in (3) is calculated as $\theta^{*}=0$, then the contrasts $Y-\theta^{*}=Y$ are equal to: $Y=0$ (51.2\% runs), $Y=1$ (38.4\% runs), $Y=2$ (9.6\% runs), $Y=3(0.8 \%$ runs $)$. The value of $(3)$ is $\psi(0)=(1 \times 0.384+$ $2 \times 0.096+3 \times 0.008) \times 0.5=0.3$. The value of $(4)$ is $m(j)=0.2$ for each $j$ and thus the value of (5) is also 0.2. Substituting into (2) we obtain $Q_{i}=(0.3-0.2) / 0.3=1 / 3$, which is a result identical to SSA where $S_{i}=1 / 3$. All higher order indices are zero.

It can be noted that $20 \%$ runs of $m(j)$ in (4) has conditional quantile $\theta^{\star}(j)=1$, which occurs in those runs where the fixed value is $X_{i}(j)=1$. Simplified, fixation of $X_{i}(j)=$ 1 gives $\theta^{*}(j)=1$, fixation $X_{i}(j)=0$ gives $\theta^{*}(j)=0$. Therefore, the change of conditional quantile $\theta^{*}(j)$ is controlled only by fixing the $i^{\text {th }}$ input variable $X_{i}(j)$ without the random influence of the other input variables, which gives $Q_{1}=Q_{2}=Q_{3}=1 / 3$ and $Q_{12}=Q_{13}=Q_{23}=Q_{123}=0$. However, if $p<0.8$ then $\theta^{\star}(j)=1$ occurs in cases with fixed values $X_{i}(j)=0$ and $X_{i}(j)=1$; i.e. quantile $\theta^{*}(j)$ is not only influenced by the fixation of one input variable, but also by the values (interactions) of the remaining two input variables. These interactions give non-zero higher order indices $Q_{12}$, $Q_{13}, Q_{23}, Q_{123}$. Interactions are not present in SSA, because fixation of one input variable influences only the conditional arithmetical mean (10) without the influence of the remaining two input variables for each $p \in(0,1)$. In terms of SSA, the model (10) is perfectly additive.

Let each $x_{i}$ have a different pmf with $a_{i}<b_{i}$. Then QSA and SSA of (10) have non-zero first order sensitivity 
indices and zero higher order indices if $p_{1}+p_{2}+p_{3} \geq 2.4$ and/or $p_{1}+p_{2}+p_{3} \leq 0.6$. In general, $S_{i} \neq Q_{i}$ with the exception of the example, which is described in the preceding two paragraphs.

Let $x_{1}, x_{2}, x_{3}$ have a normal distribution with mean value $\mu$ and standard deviation $\sigma$. SSA can be calculated analytically as $S_{1}=S_{2}=S_{3}=1 / 3$ and $Q_{12}=Q_{13}=Q_{23}=Q_{123}=0$. Contrast (8) represents the variance. $S_{i}$ indicates by how much one could reduce, on average, the output variance if $X_{i}$ could be fixed (Saltelli et al., 2004). In the presented example, fixation of two input variables will, on average, result in the same reduction of the variance as would the sum of two variances with fixations of each variable individually. On the other hand, QSA (0.5-quantile) gives $Q_{1}=Q_{2}=Q_{3}=0.183, Q_{12}=Q_{13}=Q_{23}=0.056, Q_{123}=0.283$. At first glance, there is a relatively high value of index $Q_{123}=0.283$. Index $Q_{123}$ can be calculated as $Q_{123}=1$ $Q_{1}-Q_{2}-Q_{3}-Q_{12}-Q_{13}-Q_{23}$. The non-zero value of $Q_{123}$ means that the contrast (3) is not reduced only by the conditional contrasts associated with the fixation of one or two input variables. $Q_{i j k}$ indicates by how much the contrast could reduce, on average, if one could fix $X_{i}, X_{2}, X_{3}$.

Numerical results for (10) were obtained for $N=K=$ 100000 runs of LHS and analytically in the case of SSA. Knowledge related to the 0.5 -quantile can be generalized for any other quantile.

\subsection{Multiplicative model}

Let us consider the function

$$
Y=x_{1} x_{2},
$$

where $x_{1}, x_{2}$ are statistically independent random variables with standard Gauss pdf. SSA evaluates model (11) as purely interactive. The variance $V$ of output $Y$ and the Sobol' indices can be computed analytically:

$$
V=1, S_{1}=S_{2}=0, S_{12}=1 \text {. }
$$

The value of (3) is $\psi(0)=0.318$. The value of (5) is also 0.318 . Substituting into (2) we obtain $Q_{i}=(0.318-$ $0.318) / 0.318=0$. Because the second member in the numerator (6) is zero then $Q_{12}=1$. The result $Q_{1}=Q_{2}=0$, $Q_{12}=1$ is identical with the SSA result (12). Numerical results for (11) were obtained for $N=K=1000000$ runs of LHS and analytically in the case of SSA. The same result can be obtained using the function $Y=\sin \left(x_{1}\right) \sin \left(x_{2}\right)$.

\section{Global sensitivity analysis based on metamodels}

\subsection{FE model}

The FE model is described in great detail in Kala and Valeš (2017a, 2017b). Therefore, only basic information is presented here. The aim of the computational model is to study the behaviour of a hot-rolled steel beam IPN 200 at the ultimate limit state. The beam is subjected to LTB due to uniform bending moment $M$. The computational model is created in the software Ansys APDL version 13 (ANSYS, 2014) using the element SOLID185, see Figure 3.

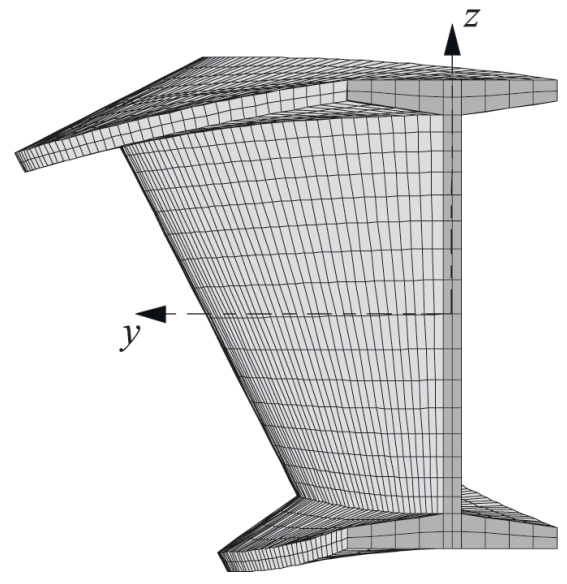

Figure 3. FE model in the Ansys program for the analysis of LTB - view in the $y-z$ plane $57 \times 58 \mathrm{~mm}(300 \times 300 \mathrm{DPI})$

End-fork boundary conditions and kinematic coupling constraints are assumed for the model. The model takes into account the influence of geometric and material imperfections, including residual stress. Residual stresses caused by rolling and cooling are considered. The residual stress distribution is implemented in the FE-model using an equivalent temperate load (Jönsson \& Stand, 2017; Kala \& Valeš, 2017a, 2017b). The magnitude of residual stresses in hot-rolled profiles is generally independent of the yield strength (Galambos, 1998; Jönsson \& Stand, 2017). The studied variable is the static resistance $R$, which is the theoretical maximum load bending moment associated with the ultimate limit state (collapse) of the beam. The calculation of $R$ is performed using the geometric and material non-linear analysis.

\subsection{Establishment of a polynomial metamodel}

QSA is evaluated using the polynomial metamodel, which was developed and described in detail in Kala and Valeš (2017a, 2017b). The metamodel approach has been shown to accelerate the evaluation of SSA (Kala \& Valeš, 2017b) or SAFD (Kala \& Valeš, 2017a) with very good efficiency.

$$
R \approx Y=\sum_{a=0}^{2} \sum_{b=0}^{2} \sum_{c=0}^{2} \sum_{d=0}^{2} \sum_{e=0}^{2} c_{\alpha} \cdot X_{1}^{a} \cdot X_{2}^{b} \cdot X_{3}^{c} \cdot X_{4}^{d} \cdot X_{5}^{e},(13)
$$

where $\alpha=3^{4} a+3^{3} b+3^{2} c+3 d+e$. Metamodel (13) replaces the highly computationally demanding FE model (Kala \& Valeš, 2017a, 2017b) based on the structural finite element SOLID185 (ANSYS, 2014) whose output is the load-carrying capacity $R$. Constants $c_{\alpha}$ are calculated for fixed $\bar{\lambda}_{L T}$ by the least square method using the LHS method and $400 \mathrm{FE}$ model runs (Kala \& Valeš, 2017a). In this article LCC is designated as resistance $R$ and 0.1 percentile of $R$ is the design resistance $R_{d}$.

The pdfs of input imperfections for steel grade S235 (Melcher, Kala, Holický, Fajkus, \& Rozlívka, 2004) are the same as in Kala and Valeš (2017a) to allow for direct comparison. Artificial random variables for the creation of the metamodel (13) are listed in Table 2 in Kala and Valeš (2017a). The real-valued random variables (random 
Table 1. Statistical characteristics of input imperfections

\begin{tabular}{|c|c|c|c|c|c|}
\hline No. & Symbol & Characteristic & Density & Mean $\mu$ & St. Deviation $\sigma$ \\
\hline 1. & $t_{2}$ & Flange thickness & Gauss & $11.3 \mathrm{~mm}$ & $0.518 \mathrm{~mm}$ \\
\hline 2. & $f_{y}$ & $\begin{array}{l}\text { Yield strength, S235 } \\
\text { Yield strength, S355 }\end{array}$ & Gauss & $\begin{array}{l}\text { 297.3 } \mathrm{MPa} \\
\text { 393.8 MPa }\end{array}$ & $\begin{array}{l}16.8 \mathrm{MPa} \\
22 \mathrm{MPa}\end{array}$ \\
\hline 3. & E & Modulus of elasticity & Gauss & $210 \mathrm{GPa}$ & $10 \mathrm{GPa}$ \\
\hline 4. & $e_{0}$ & $\begin{array}{l}\text { Initial curvature, S235 } \\
\text { Initial curvature, S355 }\end{array}$ & Gauss & $\begin{array}{l}0 \\
0\end{array}$ & $L_{1} / 1960^{\star} \quad L_{2} / 1960^{*}$ \\
\hline 5. & $r s$ & Residual stress & Gauss & $90 \mathrm{MPa}$ & $18 \mathrm{MPa}$ \\
\hline
\end{tabular}

Note: ${ }^{\star} L_{1} \approx 2.15 \bar{\lambda}_{L T}-0.75 \bar{\lambda}_{L T}^{2}+1.95 \bar{\lambda}_{L T}^{-3}-0.39 \bar{\lambda}_{L T}^{4}$ for S235, $L_{2} \approx 1.7 \bar{\lambda}_{L T}-0.36 \bar{\lambda}_{L T}^{2}+0.95 \bar{\lambda}_{L T}^{3}-0.15 \bar{\lambda}_{L T}^{4}$ for S355.

imperfections) for (13) are listed in Table 1 in Kala and Valeš (2017a) or upon expansion in Table 1 in this article.

In this article, the stochastic computational model described in Kala and Valeš (2017a) is extended to steel grade S355. With regard to the yield strength of steel S355, the statistical characteristics $\mu_{f y, S 355}=393.8 \mathrm{MPa}$, $\sigma_{f y, S 355}=22 \mathrm{MPa}$ are taken from Sadowski, Rotter, Reinke, and Ummenhofer (2015), where ten samples of steel sheets were studied. This is a relatively small sample, however, the results (Sadowski et al., 2015) are in relatively good agreement with our statistics $\mu_{f y, S 355}=394.5 \mathrm{MPa}$, $\sigma_{f y, S 355}=19.808 \mathrm{MPa}$, which were evaluated from 243 samples of flanges of profiles U65 to U140 (Melcher et al., 2008), see also discussion (Kala \& Valeš, 2018).

The FE beam model of steel S355 has two distinctions: 1$)$ the random yield strength $f_{y}$ is transformed into $f_{y}=22\left(f_{y}-297.3\right) / 16.8+393.8[\mathrm{MPa}]$ and 2$)$ the length of the beam is $L_{2} \approx 1.7 \bar{\lambda}_{L T}-0.36 \bar{\lambda}_{L T}^{2}+0.95 \bar{\lambda}_{L T}^{3}-0.15 \bar{\lambda}_{L T}^{4}$, where $L_{2}$ is also a parameter for maximum $X_{4}$ in Table 2 in Kala and Valeš (2017a).

The resistance modelling has been described in Kala and Valeš $(2017 \mathrm{a}, 2017 \mathrm{~b})$. This study focuses on issues that are specifically related to the accuracy in the tails, as it specifically influences the QSA results. The resistance from the FE model $Y_{A N S Y S}$ is calculated with an accuracy of $0.2 \%$ (Kala \& Valeš, 2017a). The accuracy of the approximation (13) is evaluated as the mean deviation $\left|Y_{\text {META }}-Y_{\text {ANSYS }}\right| / Y_{\text {ANSYS }}$, where $Y_{\text {META }}$ is resistance from metamodel (13), see Figure 4 . Columns $\Delta_{\text {all }}$ show the accuracy measured over all 400 support points. Columns $\Delta_{\text {tail }}$ show the accuracy measured over 20 smallest values of $Y_{\text {ANSYS }}$. Practically, 400 pairs $\left(Y_{\text {META }}, Y_{\text {ANSYS }}\right)$ were created, which were ranked in an ascending order according to support points $Y_{\text {ANSYS }} \Delta_{\text {tail }}$ is then evaluated for the first 20 pairs $\left(Y_{M E T A}, Y_{A N S Y S}\right)$. Accuracy $\Delta_{\text {tail }}$ is better than $\Delta_{\text {all }}$ with the exception for high slenderness values, where it is approximately the same. The accuracy of the approximation decreases with increasing slenderness of the member. The worst accuracy of the metamodel (13) is about one percent of the value $Y_{\text {ANSYS }}$, see Figure 4 .

All input random variables of the metamodel (13) are listed in Table 1 . The $r$ is value of the residual stress at edges of the flanges at any point at the midspan of the beam
(Kala \& Valeš, 2017a, 2017b). The beam is curved according to the first eigenmode. The amplitude of initial bow imperfection $e_{0}$ is located at the centre of the top flange edge at the midspan (Kala \& Valeš, 2017a, 2017b). The pdf of $e_{0}$ is symmetrical around zero and small eccentricities are more likely than large ones, although the large ones are more dangerous (Model Code, 2001). All random variables listed in Table 1 are statistically independent. A detailed explanation of the probabilistic models of all input imperfections are published in Kala and Valeš (2017a).

In this article, QSA is evaluated for 0.001-quantile (0.1 percentile) of static resistance $R$, where 0.001 -quantile represents the design resistance $R_{d}$. The calculation of $R_{d}$ is based on the semi-probabilistic approach (Freudenthal, 1956) of standard EN 1990:2002 (2003), which falls into the category of FORM methods (Sedlacek \& Müller, 2006). $R_{d}$ given as 0.1 percentile corresponds to design reliability with target reliability index of $\beta_{d}=3.8$ (failure probability $\left.P_{f}=7.2 \mathrm{E}-5\right)$ provided that we consider the ultimate limit state for common design situations within the reference period of 50 years, see Table C2 in EN 1990:2002 (2003) and/or Kala (2015).

Standard EN 1990:2002 (2003) enables the determination of design values not only from a Gauss pdf, but also from the two-parameter lognormal or Gumbel pdf, which is often assumed to reflect the effects of the random load. Resistance $R$ is often approximated using Gauss or lognormal pdfs (Model Code, 2001). In many cases very good

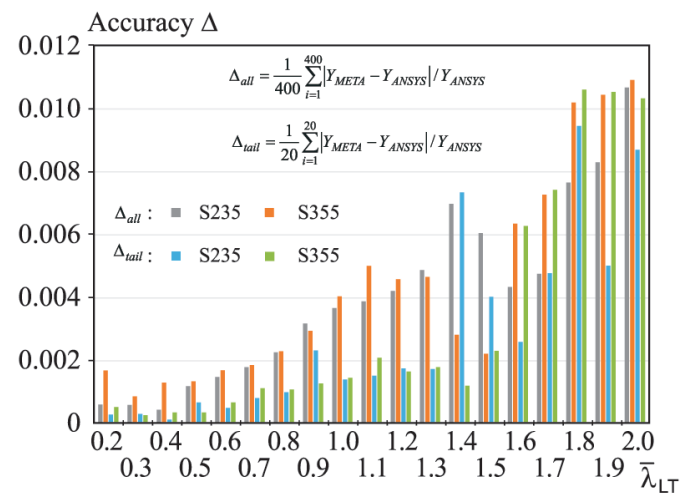

Figure 4 . The accuracy of the metamodel (13) $67 \times 49 \mathrm{~mm}$ $(300 \times 300$ DPI $)$ 
estimates of the design values of $R_{d}$ can be obtained using Hermite pdf (STATREL Manual, 1996) from Kala (2016a), which respect the skewness and kurtosis of $R$. In this article, $R_{d}$ is calculated in all cases as 0.1 percentile non-parametrically, based on LHS simulation, see Figure 1.

\subsection{Estimation of Sobol' and quantile contrast indices}

Non-dimensional slenderness $\bar{\lambda}_{L T}$ (EN 1993-1:2005, 2005 ) is a deterministic parameter that was increased using the step-by-step method with an increment of 0.01 . Two grades of structural steel S235 and S355 are considered, hence two pairs $L_{1}, L_{2}$, two stochastic computational FE models and two metamodels (13) are associated with each value $\bar{\lambda}_{L T}$. All sensitivity indices $S_{i}, S_{i j}$, etc. and $Q_{i}$, $Q_{i j}$, etc. are evaluated using the LHS method. Each sensitivity index is evaluated by double-nested-loop simulation. The same sets of (pseudo-) random numbers are used in each step, thereby ensuring that sampling and numerical errors do not swamp the result being sought (Rubinstein, 1981; Ahammed \& Melchers, 2006).

Sobol' first order sensitivity index $S_{i}(9)$ is evaluated in two cycles. Ten thousand $E\left(Y \mid X_{i}\right)$ are evaluated for each fixed value of $X_{i}$. The variance $V\left(E\left(Y \mid X_{i}\right)\right)$ is evaluated from ten thousand $E\left(Y \mid X_{i}\right)$. The unconditional variance $V(Y)$ is evaluated using 500 thousand runs of LHS. Each $S_{i}$ and 26 additional higher order Sobol' indices were evaluated with this number of runs.

The first order quantile contrast index $Q_{i}(2)$ is evaluated using $N=4000$ runs of $m(j)(4)$. Each run $m(j)$ is evaluated using a set with $K=400000$ runs of $Y$, in which $\theta^{*}(j)$ represents the conditional 0.1 -percentile evaluated as the $400^{\text {th }}$ smallest value from the same set, see Figure 1. The unconditional contrast (3) is also evaluated using $K=400000$ runs. The higher order quantile contrast indices are expressed analogously.

\section{Sensitivity analysis results}

The results of SSA depicted in Figure 5 and Figure 6 show that the results obtained for two steel grades S235 and S355 are very similar but not identical. The maximum value $S_{e 0}$ of steel S355 (red full line) is approximately $30 \%$ higher than that of steel S235 (black full line), see Figure 5. All other observations and conclusions regarding steel S355 are the same as for steel S235 and are listed in the last two chapters of Kala and Valeš (2017b). Due to the small values of the second and higher order sensitivity indices, the total sensitivity indices (Saltelli et al., 2004) were not evaluated because their size approximately corresponds to that of $S_{i}$ in Figure 7.

The plots of indices $S_{i}$ and $Q_{i}$ are approximately similar in shape; however $Q_{i}$ has much lower values than $S_{i}$. This means that QSA shows a high contribution of higher order effects (interactions). On the contrary, SSA has a relatively low share of higher order effects. For example, for $\bar{\lambda}_{L T}=0$ the size of the second order index $S_{t 2, f y}=0.44$ is almost the same as the first order index $S_{f y}=0.47$, i.e. the interaction

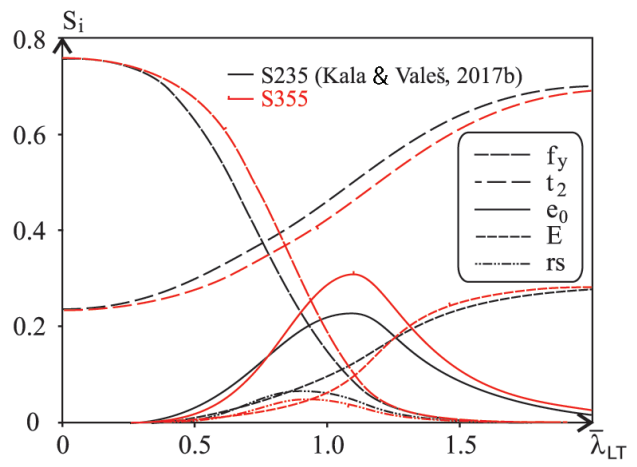

Figure 5. First order Sobol' sensitivity indices $65 \times 48 \mathrm{~mm}$ $(300 \times 300 \mathrm{DPI})$

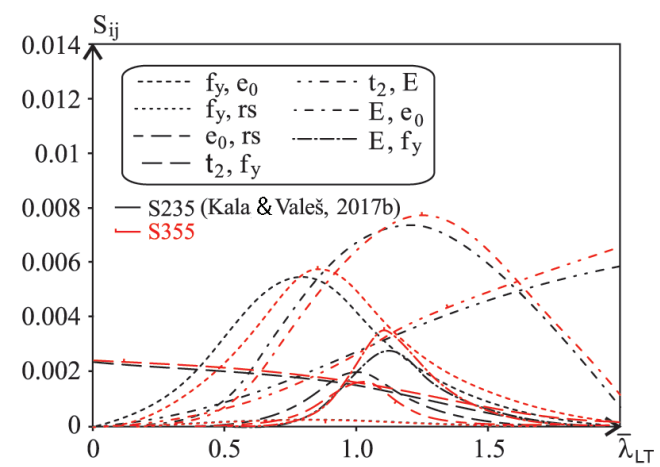

Figure 6. Second order Sobol' sensitivity indices $67 \times 47 \mathrm{~mm}$ $(300 \times 300$ DPI $)$

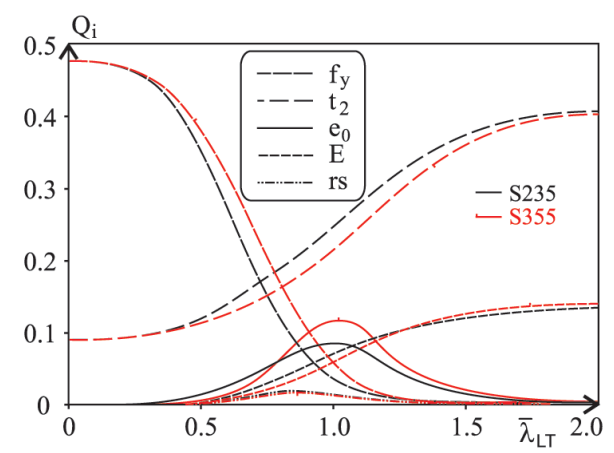

Figure 7. First order quantile contrast indices $62 \times 46 \mathrm{~mm}$ $(300 \times 300 \mathrm{DPI})$

effect of yield strength and the flange thickness is as significant as the individual effect of the yield strength. Since $Q_{i}$ are relatively small, it is not possible to deduce their sole influence on $R_{d}$, but it is necessary to study all higher order quantile contrast indices.

Figures 8 to Figure 10 show only the quantile contrast indices greater than 0.04 . The plots of indices $Q_{i j}$ and $Q_{i j k}$ show that interactions with yield strength $f_{y}$ are especially significant for beams with low and intermediate slenderness, approximately for $\bar{\lambda}_{L T}<1.2$, see Figure 8 and Figure 9. On the other hand, interactions with Young's modulus $E$ are predominantly found in beams with higher slenderness, approximately for $\bar{\lambda}_{L T}>1.2$.

Variables $f_{y}$ and $E$ do not have common second-order interactions since $Q_{f y, E} \approx 0$. However, this is not valid for the indices of the third $Q_{i j k}$ and fourth $Q_{i j k l}$ and fifth $Q_{i j k l m}$ 


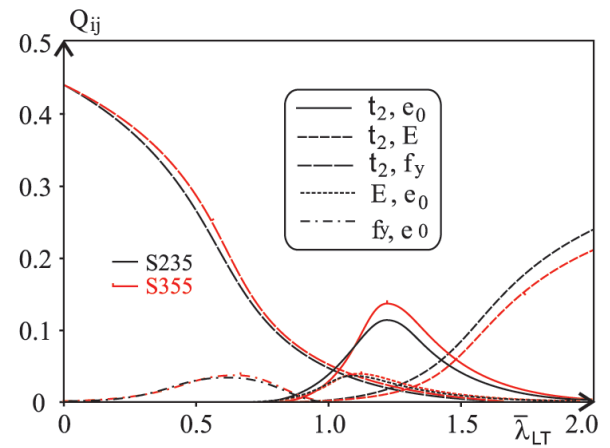

Figure 8. Second order quantile contrast indices $62 \times 46 \mathrm{~mm}$ $(300 \times 300$ DPI $)$

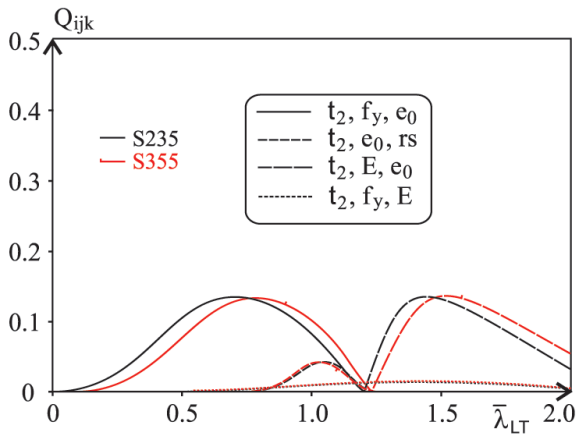

Figure 9. Third order quantile contrast indices $62 \times 46 \mathrm{~mm}$ $(300 \times 300 \mathrm{DPI})$

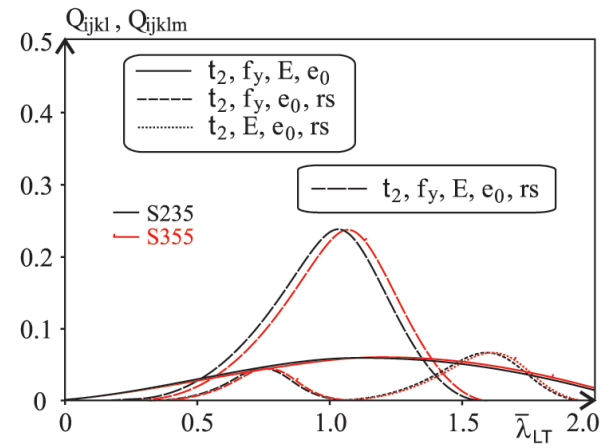

Figure 10. Fourth and fifth order quantile contrast indices $62 \times 46 \mathrm{~mm}(300 \times 300 \mathrm{DPI})$

order in which $f_{y}$ and $E$ have common interaction effects across the whole spectrum of analysed slenderness values, see plots $Q_{t 2, f y, E}$ in Figure 9 and $Q_{t 2, f y, E, e 0}$ and $Q_{t 2, f y, E, e 0, r s}$ in Figure 10.

The flange thickness $t_{2}$ is involved in all key interaction effects of any order; see Figure 8 to Figure 10. Imperfection $e_{0}$ is involved in all key interaction effects of the third and higher orders shown in Figure 9 to Figure 10, but only in one interaction index of the second order $S_{t 2, e 0}$ in Figure 8.

Due to strong interactions between imperfections, the role of each imperfection must be assessed due to its first order component and/or its interactions with the other imperfections. One possibility is to introduce the so-called total effect $S_{T i}$, which measures the first and higher order effects (interactions) of variable $X_{i}$. For example, the total effect for imperfection $e_{0}$ (fourth variable in Table 1) can

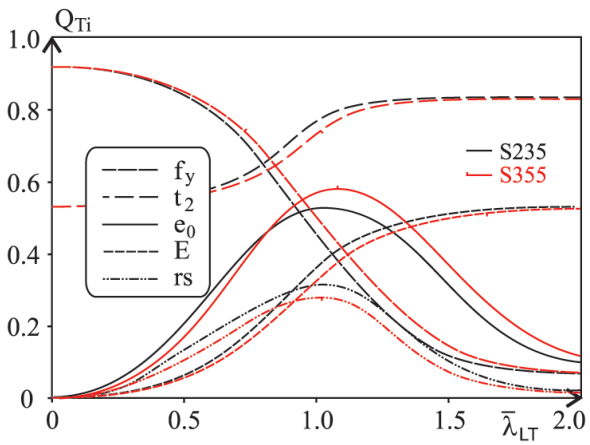

Figure 11. Total quantile contrast indices $62 \times 46 \mathrm{~mm}$ $(300 \times 300$ DPI $)$

be expressed as $Q_{T e 0}=Q_{T 4}=Q_{4}+Q_{14}+Q_{24}+Q_{34}+Q_{45}+$ $Q_{124}+Q_{134}+Q_{145}+Q_{234}+Q_{245}+Q_{345}+Q_{1234}+Q_{1245}+$ $Q_{1345}+Q_{2345}+Q_{12345}$. The total quantile contrast index for $X_{i}$ can be expressed as:

$$
Q_{T i}=1-\frac{\min _{\theta} \psi(\theta)-E\left(\min _{\theta} E\left(\psi(Y, \theta) \mid X_{\sim i}\right)\right)}{\min _{\theta} \psi(\theta)} .
$$

$Q_{T i}-Q_{i}$ is a measure of how much $X_{i}$ is involved in interactions with any other input variable. $Q_{T i}=0$ implies that $X_{i}$ is not-influential and can be fixed anywhere in its distribution without affecting the quantile $R_{d}$. The calculation methodology and LHS sample sizes $K, N$ are the same as was described in previous chapters.

The plots of $Q_{T i}(14)$ are shown in Figure 11. Proportions $Q_{T i}$ differ from proportions $Q_{i}$ (Figure 7) or $S_{i}$ (Figure 5), therefore, it can be concluded that the random variability of imperfection has a different effect on the uncertainty of the design quantile $R_{d}$ than it has on the random resistance $R$ of the structure. It is apparent from Figure 11 that imperfections $e_{0}$ and $r s$ are considerably more important than could be inferred from the individual plots of $Q_{i}$ or $S_{i}$.

\section{Conclusions}

The presented numerical study identified general agreements and significant differences between established Sobol' sensitivity analysis (SSA) oriented to mean (central parameter) of random resistance $R$ and quantile-oriented sensitivity analysis (QSA) of design resistance $R_{d}(0.1$ percentile of $R$ ).

The results of QSA and SSA are very different in the contribution of second and higher order sensitivity indices. QSA showed significant influences of interaction effects on $R_{d}$, which is in high contrast with the small interaction effects measured by classical SSA oriented on mean of $R$.

In contrast, the upward and downward trends of the first order indices $S_{i}$ and $Q_{i}$ are in general agreement; see Figure 5 and Figure 7 . The yield strength $f_{y}$ is dominant for $\bar{\lambda}_{L T}=0$. The influence of $f_{y}$ decreases and the influence of $t_{2}$ and $E$ increases with increasing slenderness. QSA and SSA exhibit a general agreement in identifying $f_{y}$ and $t_{2}$ as dominant 
imperfections, both in terms of first order indices and total indices. Imperfections $e_{0}$ and $r s$ have some influence only for intermediate slenderness. The order of the most relevant variables is the same for low and high slenderness. However, for some intermediate slenderness, the order of the second and third crucial imperfection is slightly different.

Numerical results demonstrate that both QSA and SSA are in agreement in identifying the non-influential imperfections. All indices of all orders associated with $E, e_{0}, r s$ are zero for $\bar{\lambda}_{L T}=0$, see Figure 5, Figure 6 and Figure 11 . All higher order indices related with $f_{y}$ are very small for $\lambda_{L T}=2$, however, they do not have to be zero.

The example shows that it is appropriate to analyse the effects of imperfections on the quantile $R_{d}$ using the socalled total effect $Q_{T i}$, which measures both the individual effect of input imperfection $X_{i}$ and the interaction effects among $X_{i}$ and other input imperfections. Generally, the QSA results (represented by the total indices) can change the order of importance of input variables of stochastic models aimed at verifying standard reliability indices.

The total effects of imperfections $e_{0}$ and $r s$ on the design quantile $R_{d}$ studied by QSA are much higher (see Figure 11) than those measured by the established SSA (see Figure 5 and 6). The results of QSA show that the random variability of imperfections can have a different impact on the uncertainty of the design quantiles than it has on the random response of the structure. In order to verify the design reliability represented by $R_{d}$ it is necessary to determine the statistical characteristics and pdfs of $e_{0}$ and $r s$ with greater precision than is shown by their impact on $R$ identified by SSA.

QSA is proving to be a useful tool in identifying the influence of the uncertainty of input variables on the uncertainty of design ( $0.1 \%$ quantile) and characteristic (5\% quantile) values, which deserves much more work in order to make QSA a useful and practical tool. In reliability tasks of non-linear structural mechanics with non-additive stochastic models we can expect the presence of higher order effects, which are can be appropriately described using the total quantile contrast index $Q_{T i}$. Since uncertainty is most effectively reduced by using parameters that are associated with the highest value of global importance, acquiring information on these parameters would reduce uncertainty most effectively.

QSA can re-evaluate and change views on the importance of imperfections in terms of their impact on the reliability of design provided by the EUROCODE design standards. New findings can be expected for frame structures in which SSA has shown strong stochastic interactions (Kala, 2016b). New interpretations of QSA results should be sought in the theory of structural reliability, which will address the reliability of structures.

Generally, contrast functions are a very powerful tool for estimating various parameters associated with probability distributions. By selecting the appropriate contrast function we can apply probabilistic oriented sensitivity analysis (PSA), which may be very useful in probabilistic analyses of structural reliability. New applications of QSA or PSA can be expected not only in field of technical sciences, but also in medical, biological and social sciences or the sciences of inanimate nature.

\section{Acknowledgements}

The article was elaborated within the framework of project GAČR 18-13212S. Author thanks Ing. Jan Valeš, PhD for cooperation with Ansys software.

\section{Funding}

This work was supported by the research project of the Czech Republic 18-13212S.

\section{Disclosure statement}

Authors have not any competing financial, professional, or personal interests from other parties.

\section{References}

Ahammed, M., \& Melchers, R. E. (2006). Gradient and parameter sensitivity estimation for systems. Reliability Engineering and System Safety, 91(5), 594-601.

https://doi.org/10.1016/j.ress.2005.04.005

ANSYS. (2014). ANSYS theory release 15.1.

Antucheviciene, J., Kala, Z., Marzouk, M., \& Vaidogas, E. R. (2015). Solving civil engineering problems by means of fuzzy and stochastic MCDM methods: Current state and future research. Mathematical Problems in Engineering, Article ID 362579. https://doi.org/10.1155/2015/362579

Borgonovo, E. (2007). A new uncertainty importance measure. Reliability Engineering and System Safety, 92(6), 771-784. https://doi.org/10.1016/j.ress.2006.04.015

Browne, T., Fort, J. C., Iooss, B., \& Gratiet, L. L. (2017). Estimate of $\mathrm{Q}$ uantile-oriented sensitivity indices. Preprint. Retrieved from https://hal.archives-ouvertes.fr/hal-01450891

Chalmovsky, J., Stefanak, J., Mica, L., Kala, Z., Skudois, S., Norkus, A., \& Zilioniene, D. (2017). Statistical-numerical analysis for pullout tests of ground anchors. The Baltic Journal of Road and Bridge Engineering, 12(3), 145-153. https://doi.org/10.3846/bjrbe.2017.17

D’Angelo, L., \& Nussbaumer, A. (2017). New framework for calibration of partial safety factors for fatigue design. Journal of Constructional Steel Research, 139, 466-472.

https://doi.org/10.1016/j.jcsr.2017.10.006

European Committee for Standardization. (2003). Eurocode: Basis of structural design (EN 1990:2002). Brussels. Retrieved from https://www.unirc.it/documentazione/materiale_didattico/599_2010_260_7481.pdf

European Committee for Standardization. (2005). Eurocode 3: Design of steel structures - Part 1: General rules and rules for buildings (EN 1993-1:2005). Retrieved from https://eurocodes.jrc.ec.europa.eu/showpage.php?id=133

Fort, J. C., Klein, T., \& Rachdi, N. (2016). New sensitivity analysis subordinated to a contrast. Communications in Statistics Theory and Methods, 45(15), 4349-4364. https://doi.org/10.1080/03610926.2014.901369

Freudenthal, A. M. (1956). Safety and the probability of structural failure. American Society of Civil Engineers Transactions, 121, 1337-1397.

Galambos, T. V. (1998). Guide to stability design criteria for metal structures (5 $5^{\text {th }}$ ed.). Wiley. 
Hariri-Ardebili, M. A., \& Pourkamali-Anaraki, F. (2018). Simplified reliability analysis of multi hazard risk in gravity dams via machine learning techniques. Archives of Civil and Mechanical Engineering, 18(2), 592-610. https://doi.org/10.1016/j.acme.2017.09.003

Iman, R. L., \& Conover, W. J. (1980). Small sample sensitivity analysis techniques for computer models. With an application to risk assessment. Communications in Statistics - Theory and Methods, 9(17), 1749-1842.

https://doi.org/10.1080/03610928008827996

Jönsson, J., \& Stand, T. C. (2017). European column buckling curves and finite element modelling including high strength steels. Journal of Constructional Steel Research, 128, 136-151. https://doi.org/10.1016/j.jcsr.2016.08.013

Kala, Z. (2012). Geometrically non-linear finite element reliability analysis of steel plane frames with initial imperfections. Journal of Civil Engineering and Management, 18(1), 81-90. https://doi.org/10.3846/13923730.2012.655306

Kala, Z. (2015). Reliability analysis of the lateral torsional buckling resistance and the ultimate limit state of steel beams with random imperfections. Journal of Civil Engineering and Management, 21(7), 902-911. https://doi.org/10.3846/13923730.2014.971130

Kala, Z. (2016a). Global interval sensitivity analysis of hermite probability density function percentiles. International Journal of Mathematical Models and Methods in Applied Sciences, 10, 373-380.

Kala, Z. (2016b). Global sensitivity analysis in stability problems of steel frame structures. Journal of Civil Engineering and Management, 22(3), 417-424. https://doi.org/10.3846/13923730.2015.1073618

Kala, Z. (2018). Benchmark of goal oriented sensitivity analysis methods using Ishigami function. International Journal of Mathematical and Computational Methods, 3, 43-50.

Kala, Z., \& Valeš, J. (2017a). Sensitivity assessment and lateraltorsional buckling design of I-beams using solid finite elements. Journal of Constructional Steel Research, 139, 110-122. https://doi.org/10.1016/j.jcsr.2017.09.014

Kala, Z., \& Valeš, J. (2017b). Global sensitivity analysis of lateraltorsional buckling resistance based on finite element simulations. Engineering Structures, 134, 37-47. https://doi.org/10.1016/j.engstruct.2016.12.032

Kala, Z., \& Valeš, J. (2018). Imperfection sensitivity analysis of steel columns at ultimate limit state. Archives of Civil and Mechanical Engineering, 18(4), 1207-1218. https://doi.org/10.1016/j.acme.2018.01.009

Kala, Z., Valeš, J., \& Jönsson, J. (2017). Random fields of initial out of straightness leading to column buckling. Journal of Civil Engineering and Management, 23(7), 902-913. https://doi.org/10.3846/13923730.2017.1341957

Kucherenko, S., Song, S., \& Wang, L. (2019). Quantile based global sensitivity measures. Reliability Engineering and System Safety, 185, 35-48. https://doi.org/10.1016/j.ress.2018.12.001

Li, L., Lu, Z., Zhang, K., \& Gao, Q. (2017). General validation and decomposition of the variance-based measures for models with correlated inputs. Aerospace Science and Technology, 62, 75-86. https://doi.org/10.1016/j.ast.2016.12.003

Liu, C., He, L., Yhenyu, W., \& Yuan, J. (2018). Experimantal study on joint stiffness with vision-based system and geometric imperfections of temporary member structure. Journal of Civil Engineering and Management, 24(1), 43-52. https://doi.org/10.3846/jcem.2018.299

Massart, P. (2003). Concentration ineQualities and model selection. New York: Springer.
Maume-Deschamps, V., \& Niang, I. (2018). Estimation of quantile oriented sensitivity indices. Statistics and Probability Letters, 134, 122-127. https://doi.org/10.1016/j.spl.2017.10.019

McKey, M. D., Beckman, R. J., \& Conover, W. J. (1979). A comparison of the three methods of selecting values of input variables in the analysis of output from a computer code. Technometrics, 21(2), 239-245.

Melcher, J., Kala, Z., Holický, M., Fajkus, M., \& Rozlívka, L. (2004). Design characteristics of structural steels based on statistical analysis of metallurgical products. Journal of Constructional Steel Research, 60(3-5), 795-808. https://doi.org/10.1016/S0143-974X(03)00144-5

Melcher, J., Kala, Z., Karmazínová, M, Fajkus, M., Holický, M., Rozlívka, L., \& Puklický, L. (2008). Statistical evaluation of material characteristics and their influence on design strength of structural steel of S355. In Proceedings of the $5^{\text {th }}$ European Conference on Steel and Composite Structures, Research - Practice New materials (Eurosteel 2008) (pp. 809-814). Graz, Austria.

Model Code. (2001). Joint Committee of Structural Safety (JCSS). Retrieved from http://www.jcss.ethz.ch

Rachdi, N. (2011). Apprentissage statisti $\mathrm{Q}$ ue et computer experiments - Approche $\mathrm{Quantitative} \mathrm{du} \mathrm{ris} \mathrm{Que}$ et des incertitudes en modélisation ( $\mathrm{PhD}$ thesis, Université Toulouse). Retrieved from http://thesesups.ups-tlse.fr/1538/1/2011TOU30283.pdf

Rubinstein, R. Y. (1981). Guide to stability design criteria for metal structures. New York: John Wiley and Sons.

Sadowski, A., Rotter, J. M., Reinke, T., \& Ummenhofer, T. (2015). Statistical analysis of the material properties of selected structural carbon steels. Structural Safety, 53, 26-35. https://doi.org/10.1016/j.strusafe.2014.12.002

Saltelli, A., Chan, K., \& Scott, E. M. (2004). Sensitivity analysis. Wiley series in probability and statistics. New York: John Wiley and Sons.

Sedlacek, G., \& Kraus, O. (2007). Use of safety factors for the design of steel structures according to the Eurocodes. Engineering Failure Analysis, 14(3), 434-441. https://doi.org/10.1016/j.engfailanal.2005.08.002

Sedlacek, G., \& Müller, C. (2006). The European standard family and its basis. Journal of Constructional Steel Research, 62(11), 1047-1059. https://doi.org/10.1016/j.jcsr.2006.06.027

Sobol', I. M. (1993). Sensitivity analysis for non-linear mathematical models. Mathematical Modelling and Computational Experiment, 1, 407-414. [Translated from Russian: I. M. Sobol'. 1990. Sensitivity estimates for nonlinear mathematical models. Matematicheskoe Modelirovanie, 2, 112-118].

Sobol', I. M. (2001). Global sensitivity indices for nonlinear mathematical models and their Monte Carlo estimates. Mathematics and Computers in Simulation, 55(1-3), 271-280. https://doi.org/10.1016/S0378-4754(00)00270-6

STATREL Manual. (1996). Statistical analysis of data for reliability applications. Version: 3.10.

Vales, J., \& Stan, T. C. (2017). FEM modelling of lateral-torsional buckling using shell and solid elements. Procedia Engineering, 190, 464-471. https://doi.org/10.1016/j.proeng.2017.05.365

Vapnik, V. N. (1998). Statistical learning theory. New York: John Wiley and Sons.

Xiao, S., Lu, Z., \& Wang, P. (2018). Global sensitivity analysis based on distance correlation for structural systems with multivariate output. Engineering Structures, 167, 74-83. https://doi.org/10.1016/j.engstruct.2018.04.027

Zhang, K., Lu, Z., Wu, D., \& Zhang, Y. (2017). Analytical variance based global sensitivity analysis for models with correlated variables. Applied Mathematical Modelling, 45, 748-767. https://doi.org/10.1016/j.apm.2016.12.036 